\title{
Quality and learning curve of handheld versus stand-alone non-mydriatic cameras
}

\author{
This article was published in the following Dove Press journal: \\ Clinical Ophthalmology \\ 31 August 2017 \\ Number of times this article has been viewed
}

\author{
Mariya Gosheva' \\ Christian Klameth' \\ Lars Norrenberg ${ }^{2}$ \\ Lucien $\mathrm{Clin}^{3}$ \\ Johannes Dietter ${ }^{4}$ \\ Wadood $\mathrm{Haq}^{4}$ \\ Iliya $\mathrm{V}$ Ivanov ${ }^{4-6}$ \\ Focke Ziemssen' \\ Martin A Leitritz ${ }^{7}$ \\ 'University Eye Hospital, Centre \\ for Ophthalmology, Tuebingen, \\ ${ }^{2}$ Department of Obstetrics and \\ Gynecology, Klinikum am Steinenberg, \\ District Hospital Reutlingen, \\ Reutlingen, ${ }^{3}$ School of Informatics, \\ Reutlingen University, ${ }^{4}$ Division \\ of Experimental Ophthalmology, \\ Centre for Ophthalmology, Institute \\ for Ophthalmic Research, ${ }^{5}$ Vision \\ Rehabilitation Research Unit, Centre \\ for Ophthalmology, University Eye \\ Hospital, Eberhard Karls, ${ }^{6}$ ZEISS Vision \\ Science Lab, Institute for Ophthalmic \\ Research, Centre for Ophthalmology, \\ University of Tuebingen, ${ }^{7}$ Section for \\ Experimental Ophthalmic Surgery \\ and Refractive Surgery, University Eye \\ Hospital, Centre for Ophthalmology, \\ Tuebingen, Germany
}

Correspondence: Christian Klameth University Eye Hospital, Centre for Ophthalmology, Tuebingen, Germany

Tel +49 1733548039

Email c.klameth@gmx.de
Purpose: Nowadays, complex digital imaging systems allow detailed retinal imaging without dilating patients' pupils. These so-called non-mydriatic cameras have advantages in common circumstances (eg, for screening or emergency purposes) but present limitations in terms of image quality and field of view. We compare the usefulness of two non-mydriatic camera systems (ie, a handheld versus a stand-alone device) for fundus imaging. The primary outcome was image quality. The secondary outcomes were learning effects and quality grade-influencing factors. Methods: The imaging procedures followed standard protocol and were all performed by the same investigator. Camera $1\left(\mathrm{DRS}^{\circledR}\right)$ was a stand-alone system, while Camera 2 (Smartscope ${ }^{\circledR}$ PRO) was a mobile system. In order to evaluate possible learning effects, we selected an examiner with no prior training in the use of these systems. The images were graded separately by two experienced and "blinded" ophthalmologists following a defined protocol.

Results: In total, 211 people were enrolled. Quality grade comparisons showed significantly better grades for Camera 1. Both systems achieved better quality grades for macular images than for disc-centered images. No remarkable learning effects could be demonstrated.

Conclusions: Both camera systems are useful for fundus imaging. The greater mobility of Camera 2 was associated with lower image quality. For screening scenarios or telemedicine, it must be determined whether image quality or mobility is more important.

Keywords: imaging, non-mydriatic, handheld camera, learning curve

\section{Introduction}

In 1887, Lucien Howe described an experimental approach for fundus imaging in his publication "Photography of the Interior of the Eye". ${ }^{1}$ In the following decades, increased light sensitivity, optimized optics, new illumination sources and digital techniques led to a continuous increase in the image quality. ${ }^{2}$ Today, state-of-the-art digital and complex imaging systems (cameras and scanners) allow detailed imaging of retinal structures without dilating patients' pupils. ${ }^{3}$ Although non-mydriatic cameras provide partially reduced image quality and a reduced field of view compared to conventional cameras, the former have advantages in common circumstances (eg, for screening or emergency purposes). ${ }^{4-7}$

The present study compares the usefulness of two non-mydriatic camera systems (ie, a handheld versus a stand-alone device) for fundus imaging needs. The primary outcome was image quality. The secondary outcomes were learning effects and quality grade-influencing factors.

\section{Methods}

People willing to be part of the study were randomly included after providing informed consent. The only other inclusion criteria were that participants must be at 
least 18 years of age. In addition to patients of the hospital, staff and patients' companions could also be included. Each person underwent imaging in a completely dark room with both camera systems, in a randomly assigned chronology of the latter. Before taking the first images (right eye first, left eye second) with each camera system, a dark adaptation time of 2 minutes was allowed.

Camera 1 (DRS ${ }^{\circledR}$, software version: 2.2.11; CenterVue $\mathrm{SpA}$, Padua, Italy) is a stand-alone system and uses a field of view of $45 \times 40^{\circ}$ with a 5 -megapixel sensor with a resolution of 2,592 $\times 1,944$ pixels. Camera 1 was mounted on a mobile lifting table; the patients sat on a vertically adjustable chair. Similar to the most diagnostic machinery in ophthalmology, Camera 1 had a chinrest and a forehead bar to allow for optimized positioning of the patient's head. Thus, the camera lens faced toward the patient while the rear part of the camera, where the operating display was located, faced toward the physician. The pause time between each photograph was adjusted to 40 seconds with an integrated timer to allow for sufficient pupil relaxation after the camera flash, in accordance with comparable studies with non-mydriasis camera systems. $^{4}$

Camera 2(Smartscope ${ }^{\circledR}$ PRO, software version: 3.2.6.3498; Optomed Oy, Oulu, Finland) is a handheld system that uses a field of view of $40 \times 40^{\circ}$ with a 5 -megapixel sensor with a resolution of $1,536 \times 1,152$ pixels. The small on-device display can be used for aiming and quick quality control of the pictures taken. Camera 2 was used in a handheld setting with the patient and the physician stably positioned and sitting face to face, with the patient holding the camera system. The pause time between each photograph was adjusted to 40 seconds and measured with the help of a stopwatch.

With both camera systems, we aimed to capture, for each person, first an optic disc-centered image and then a maculacentered image (first of the right eye, then of the left eye). The image files of both systems were temporarily saved on internal storage and later copied for further evaluation using an external hard drive. In cases of bad image quality, one further attempt at increased quality was made by retaking the images.

Prior to this study, the examiner was an untrained medical doctor (LN) who read the manufacturer's instructions for each imaging device and underwent a theoretical briefing from an experienced ophthalmologist (MAL) followed by a practical session with an examination of three patients for training purposes (six eyes).

The images were graded separately by two experienced and "blinded" ophthalmologists as subinvestigators (MG, CK)
Table I Quality grade classification

\begin{tabular}{ll}
\hline Grade & Description \\
\hline I & Ideal quality \\
2 & Subtle findings can be excluded; image quality not ideal \\
3 & Obvious emergent findings can be excluded, no details \\
4 & Excluding all emergency findings is not possible \\
5 & Inadequate for any evaluation \\
\hline
\end{tabular}

at the Tübingen Eye Reading Center using calibrated 27 " in-plane switching displays with full high-definition resolution and 32-bit color depth. The quality grading (Table 1; Figure 1) of an image was performed using a system, such as the 5-point scale of Lamirel et al. ${ }^{4}$

So that the results in the first step could be compared, all of the images taken were included. In the second step, only images from the participants' right eyes were used, thus minimizing bias based on the reduction of pupil sizes after several photographs and the accompanying camera flashes. The right eye image selection is designated as "selection OD" (sOD) in the following text.

The collected data were descriptively analyzed using a statistical program (JMP 11.1; SAS Institute, Cary, NC, USA). All data were anonymously analyzed in accordance with the German directives on information security and data protection.

Written informed consent was obtained from all patients before the examination. The investigation followed the tenets of the Declaration of Helsinki. Approval from the responsible ethics committees (Ethics committee of the Medical Faculty, Eberhard Karls University, Tübingen; Ethics committee of the Medical Association Baden-Württemberg, Stuttgart) was obtained in advance. The study is registered at ClinicalTrials.gov (ID NCT02089009).

\section{Results}

In total, 211 (male: 35, female: 176) people were enrolled in the study. The median age was 35 years (minimum: 18 years, maximum: 66 years). The comparison of the achieved image quality grade for both camera systems was subdivided first for all images (Figure 2; Table 2; and for sOD only, Table 3). Fewer images were taken with Camera 2 than with Camera 1 . This was due to a temporary mechanical defect within the period of data acquisition.

Quality grade comparisons were performed using the Wilcoxon/Kruskal-Wallis test and showed significantly lower (better) quality grades for all images and for sOD for Camera 1. Both systems achieved better quality grades for macular images than for disc-centered images. 


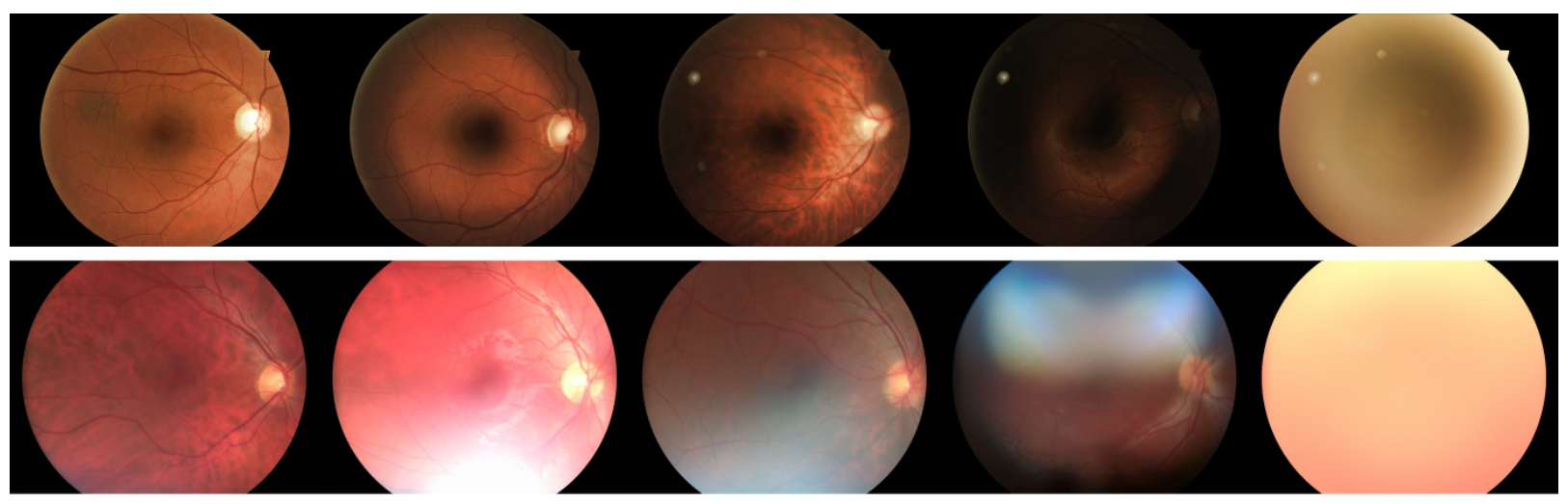

Figure I Image quality examples, beginning with grade I on the left side and ending with grade 5 on the right side. Note: The upper row is from Camera I and the lower row is from Camera 2.

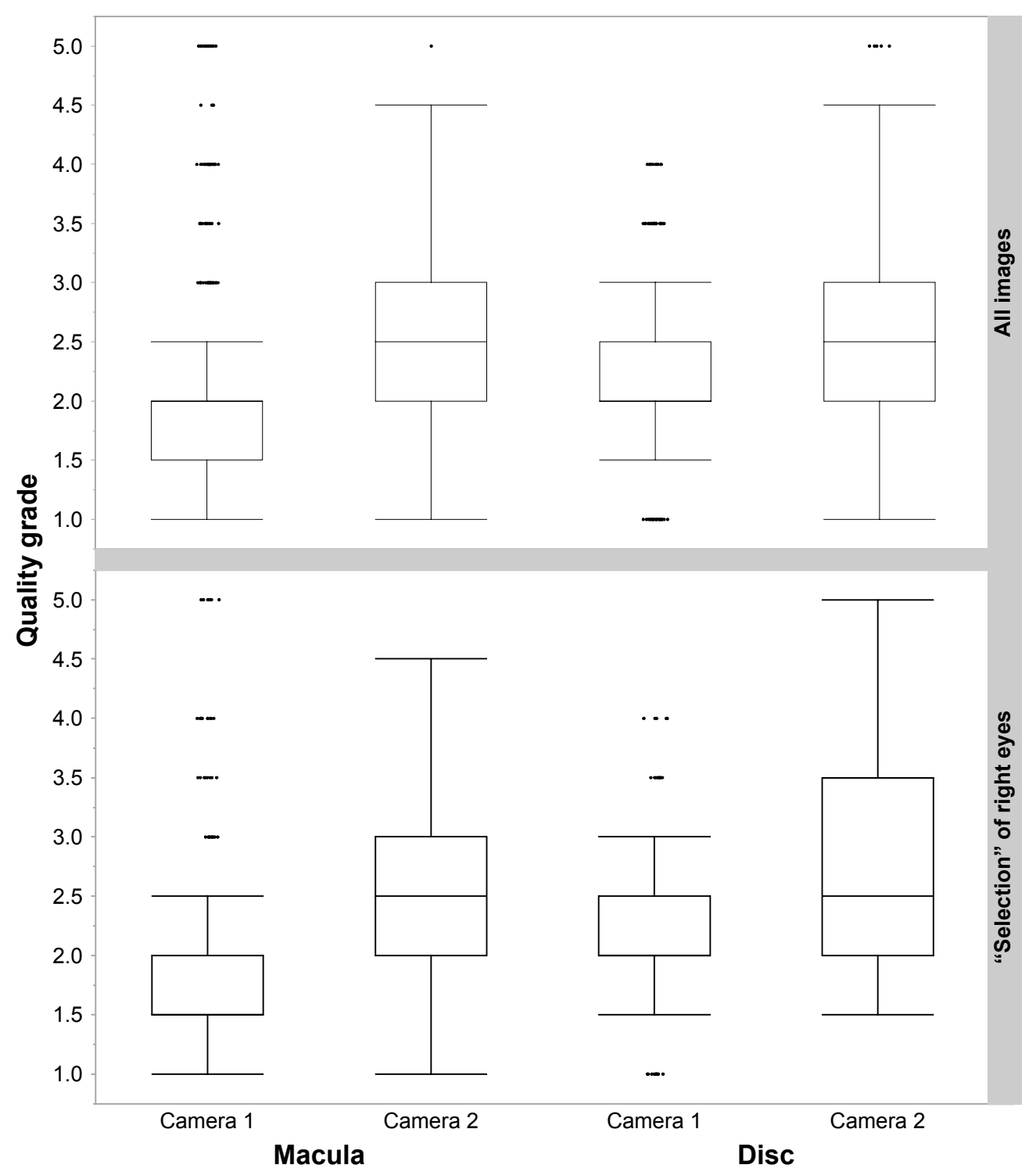

Figure 2 Achieved quality grade ( $y$-axis) for all images (upper panel) and sOD images (lower panel) divided into regions of interest, that is, macula versus disc ( $x$-axis) and camera system (x-axis).

Note: Box and whisker diagrams show $5 \%$ and $95 \%$ quantiles (whiskers) and $25 \%$ and $75 \%$ quantiles (box).

Abbreviation: SOD, selection OD. 
Table 2 Descriptive overview of all evaluated images for both camera systems

\begin{tabular}{|c|c|c|c|}
\hline Parameter & Camera I & Camera 2 & \\
\hline Images total & $\mathrm{I}, 044$ & 873 & \\
\hline Macula-centered & 541 & 433 & \\
\hline Disc-centered & 503 & 440 & \\
\hline Quality of macula-centered images, mean (SD) & $1.95(0.93)$ & $2.61(0.68)$ & $P<0.000$ I (Wilcoxon/Kruskal-Wallis test) \\
\hline Quality of disc-centered images, mean (SD) & $2.21(0.65)$ & $2.69(0.81)$ & $P<0.000$ I (Wilcoxon/Kruskal-Wallis test) \\
\hline Macula-centered images with quality grade 1 or 2 & $484(89 \%)$ & $385(83 \%)$ & \\
\hline Disc-centered images with quality grade 1 or 2 & 464 (92\%) & $343(78 \%)$ & \\
\hline
\end{tabular}

Furthermore, we evaluated the quality grading regarding learning effects of the used camera systems. Within the first 100 patients, we could not detect any significant learning effects. The highest increase in image quality could be seen as a trend for disc-centered images of Camera 2 (Figure 3).

Logistic regression analyses were performed to evaluate the factors influencing the quality grade for sOD. The camera system resulted in a regression coefficient of 1.56 (standard error [SE]: 0.16), the image area in a regression coefficient of -0.28 (SE: 0.12), and the first-used camera device in a regression coefficient of 0.144 (SE: 0.20 ).

The internal measurement (Camera 1) of pupil diameter for sOD resulted in a mean diameter of $3.763 \mathrm{~mm}$ (SD: 0.73). A logistic regression analysis for Camera $1 \mathrm{sOD}$ resulted in a regression coefficient for a pupil width of 2.55 (SE: 0.31). In cases in which the diameter of the pupil was $\geq 3.0 \mathrm{~mm}$, we reached, in $82 \%$ of images, a quality better than 2.5 . In cases with lower pupil width, only $25 \%$ of the images were of good quality $(<2.5)$.

\section{Discussion}

Within our study, we compared two different camera devices that are optimized for non-mydriatic imaging. The systems were used by a previously inexperienced medical doctor. Image quality grades were superior for Camera 1 compared to Camera 2 and were better for macula-centered images than for disc-centered images. There were no significant learning curves to record. To eliminate technical bias (ie, pupil diameter and flash interval), we evaluated not only all images in total but also right eye images only (sOD).
In addition to scanning laser ophthalmoscopy ${ }^{8}$ and optical coherence tomography, ${ }^{5}$ non-mydriatic photography systems are used in telemedicine and screening programs. Therefore, evaluations of usability have been performed for several systems (eg, for diabetic retinopathy screening ${ }^{9,10}$ or age-related macular degeneration screening).${ }^{11}$ Lamirel et al reported on important factors for increasing the quality of non-mydriatic photography operated by non-ophthalmically trained personnel in an emergency department. ${ }^{4}$ Despite the fact that groups of patients with, for example, age-related macular degeneration are older, we used a particularly young group of patients in order to create an optimal situation in which to compare the systems. We deemed this decision to be reasonable in this setting in order to eliminate the effects of pathology-caused influencing factors (eg, cataracts, corneal diseases, lack of concentration) on these two parameters. Still, further studies could investigate how great the effects of these factors really are on the results of each individual camera system.

We compared two independently developed camera devices that were designed for use outside an ophthalmological center. Beyond the common aim of achieving nonmydriatic photography, the systems are different with regard to their architecture. Camera 1 focuses on automation while Camera 2 aims for mobility. From our point of view, the price of increased mobility seems to be decreased stability and, therefore, reduced image quality. When the patient's pupils are not dilated, it is a challenge to keep the patient's eye and the camera aligned in terms of axis and distance (focus). Our expectation that there would be significant learning effects and an increase in image quality was not fulfilled. These

Table 3 Descriptive overview of sOD images

\begin{tabular}{llll}
\hline Parameter & Camera I & Camera 2 \\
\hline Macula-centered & 220 & 190 & 183 \\
Disc-centered & 202 & $2.57(0.69)$ & $P<0.000$ I (Wilcoxon/Kruskal-Wallis test) \\
Quality macula-centered images, mean (SD) & $1.94(0.9)$ & $2.73(0.06)$ & $P<0.000$ I (Wilcoxon/Kruskal-Wallis test) \\
Quality disc-centered images, mean (SD) & $2.15(0.64)$ & $87(39 \%)$ & $86(40 \%)$ \\
Macula-centered images with quality grade I or 2 & $222(81 \%)$ & $177(71 \%)$ & \\
Disc-centered images with quality grade I or 2 & &
\end{tabular}

Abbreviation: sOD, selection OD. 


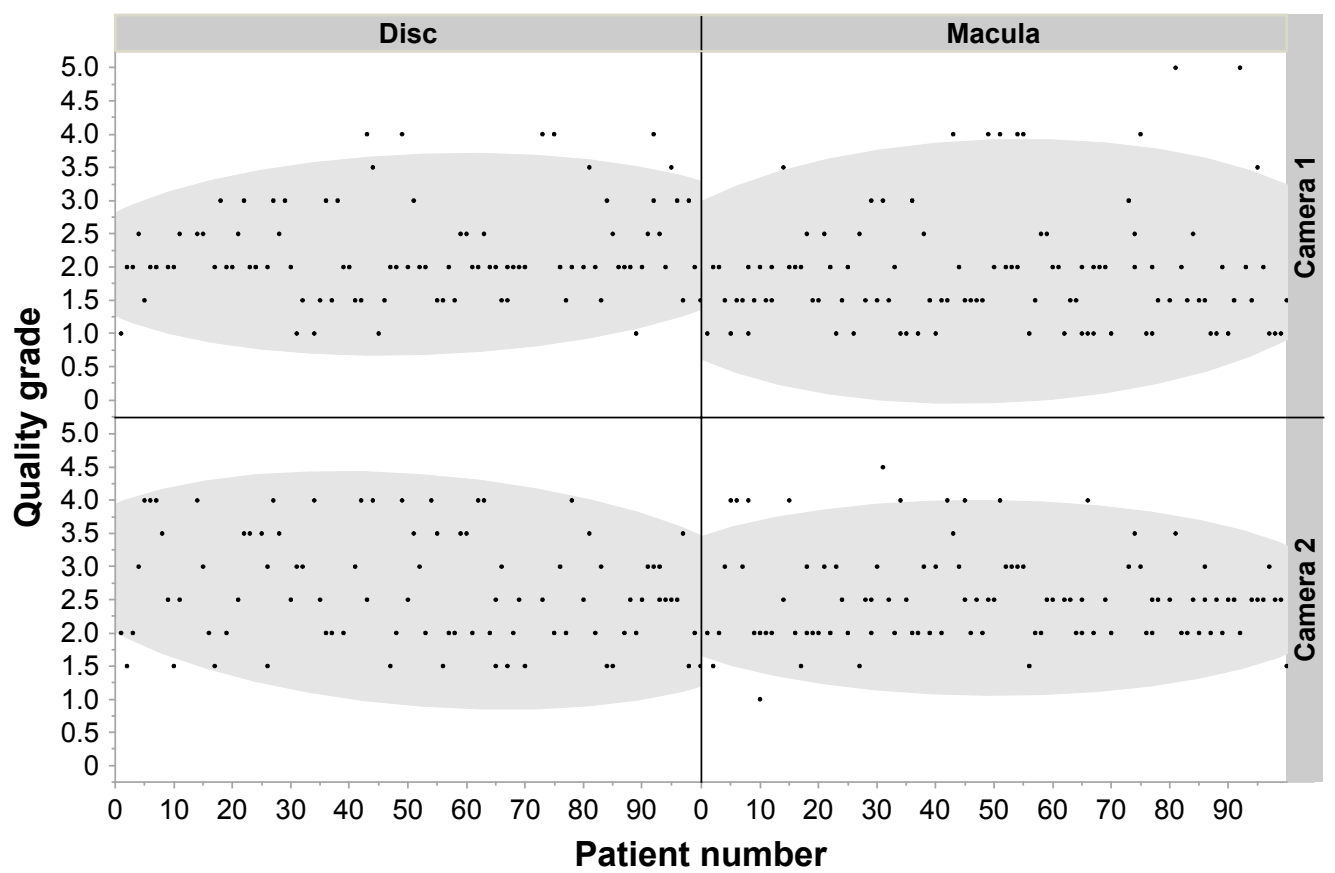

Figure 3 Achieved quality grade ( $y$-axis) of images from the right eye of each patient (x-axis) divided into regions of interest, that is, macula versus disc (upper $x$-axis) and camera system (right y-axis).

Note: The gray fields mark the $90 \%$ density ellipse.

results could change if a greater number of patients were studied. Still, factors such as anatomical limitations (eg, deep eye sockets) or physiological limitations (eg, tremors on the part of the physician or patient), which are likely to exist, especially when the patient group is not ideal, could lead to sustained image quality problems. So, the choice of which camera system is best might very much depend on the field in which the system is to be used; if the camera can be placed in a permanent position (eg, in the office of a general practitioner's private practice, in a field hospital, or in a nursing home) Camera 1 will deliver better quality images, and the price for reduced mobility seems to be acceptable. If the screening is to be performed under very mobile conditions (eg, in rural areas in developing countries, or during home visits by a physician), the ability to take an image of relatively low quality might be preferable to taking no image or to losing time by setting up a heavy device for each examination.

In 2013, Ogunyemi et al investigated the use of autonomy versus the automation of non-mydriatic systems. ${ }^{12}$ The study showed that the image quality of the automated system (which correlates to Camera 1) was obviously worse (in total, $61 \%$ of 142 images had a rating of excellent, good, or adequate) than our findings. These differences could have been caused by other imaging areas of the posterior pole and time delay protocols, but the results were not reported separately for the area subgroups. ${ }^{12}$ In addition, Ogunyemi et al reported frustration and increased wait times with the automated system. ${ }^{12}$ Within our setting, we could not confirm these findings, which could be based on differences in personnel.

The reduced quality of disc-centered images compared to macula-centered images is likely linked to the diameter of the pupil. The former is slightly tilted to image the disc, and therefore, the pass-through of light is reduced. We showed that reduced pupil diameters resulted in lower image quality for Camera 1. To get a good set of usable images, the width of the patient's pupil should be $3.0 \mathrm{~mm}$ at a minimum. For Camera 2, such diameter values were not measured within the system. A limitation of the diameter measurements of Camera 1 is that we did not perform a crosscheck of these measurements with other devices.

\section{Conclusion}

Both camera systems studied here are useful for fundus imaging. The greater mobility of Camera 2 is associated with lower image quality. For screening scenarios or telemedicine, it must be determined whether image quality or mobility is more important. The limitations of our study include the relatively young patient group and the absence of crosschecked pupil measurements with Camera 1.

\section{Acknowledgments}

Dr Leitritz received grants and non-financial support from Optomed Oy during the study. The results of the study were not influenced by any external support. 


\section{Disclosure}

The authors report no conflicts of interest in this work.

\section{References}

1. Howe L. Photography of the interior of the eye. Trans Am Ophthalmol Soc. $1887 ; 4: 568-571$.

2. Bennett TJ, Barry CJ. Ophthalmic imaging today: an ophthalmic photographer's viewpoint - a review. Clin Exp Ophthalmol. 2009;37(1): $2-13$.

3. Rasmussen ML, Broe R, Frydkjaer-Olsen U, et al. Comparison between early treatment diabetic retinopathy study 7 -field retinal photos and non-mydriatic, mydriatic and mydriatic steered widefield scanning laser ophthalmoscopy for assessment of diabetic retinopathy. J Diabetes Complications. 2015;29(1):99-104.

4. Lamirel C, Bruce BB, Wright DW, Delaney KP, Newman NJ, Biousse V. Quality of nonmydriatic digital fundus photography obtained by nurse practitioners in the emergency department: the FOTO-ED study. Ophthalmology. 2012;119(3):617-624.

5. Ouyang Y, Heussen FM, Keane PA, Sadda SR, Walsh AC. The retinal disease screening study: retrospective comparison of nonmydriatic fundus photography and three-dimensional optical coherence tomography for detection of retinal irregularities. Invest Ophthalmol Vis Sci. 2013; 54(8):5694-5700.
6. Chin EK, Ventura BV, See KY, Seibles J, Park SS. Nonmydriatic fundus photography for teleophthalmology diabetic retinopathy screening in rural and urban clinics. Telemed J E Health. 2014;20(2):102-108.

7. Mackay DD, Bruce BB. Non-mydriatic fundus photography: a practical review for the neurologist. Pract Neurol. 2016;16(5):343-351.

8. Neubauer AS, Kernt M, Haritoglou C, Priglinger SG, Kampik A, Ulbig MW. Nonmydriatic screening for diabetic retinopathy by ultrawidefield scanning laser ophthalmoscopy (Optomap). Graefes Arch Clin Exp Ophthalmol. 2008;246(2):229-235.

9. Schwartz S, Harasawa M, Baldivieso V, Sabel AL, Mandava N, Quiroz-Mercado H. Nonmydriatic fundus camera for diabetic retinopathy screening in a safety net hospital: effectiveness, prevalence, and risk factors. Eur J Ophthalmol. 2015;25(2):145-152.

10. Gupta V, Bansal R, Gupta A, Bhansali A. Sensitivity and specificity of nonmydriatic digital imaging in screening diabetic retinopathy in Indian eyes. Indian J Ophthalmol. 2014;62(8):851-856.

11. De Bats F, Vannier Nitenberg C, Fantino B, Denis P, Kodjikian L. Agerelated macular degeneration screening using a nonmydriatic digital color fundus camera and telemedicine. Ophthalmologica. 2014;231(3): 172-176.

12. Ogunyemi O, Moran E, Patty Daskivich L, et al. Autonomy versus automation: perceptions of nonmydriatic camera choice for teleretinal screening in an urban safety net clinic. Telemed JE Health. 2013;19(8): $591-596$.
Clinical Ophthalmology

\section{Publish your work in this journal}

Clinical Ophthalmology is an international, peer-reviewed journal covering all subspecialties within ophthalmology. Key topics include: Optometry; Visual science; Pharmacology and drug therapy in eye diseases; Basic Sciences; Primary and Secondary eye care; Patient Safety and Quality of Care Improvements. This journal is indexed on

\section{Dovepress}

PubMed Central and CAS, and is the official journal of The Society of Clinical Ophthalmology (SCO). The manuscript management system is completely online and includes a very quick and fair peer-review system, which is all easy to use. Visit http://www.dovepress.com/ testimonials.php to read real quotes from published authors. 